\title{
Positionspapier Magnetresonanztomografie als fachfremde Leistung
}

Die Magnetresonanztomografie (MRT) ist ein innovatives und zugleich sehr komplexes und technisch aufwendiges Verfahren in der Radiologie. Zudem können Fehler vor und während der Untersuchung oder bei der Befundung schnell zu einem Risiko für Patientinnen und Patienten führen. Für die Indikation, Durchführung und Befunderstellung von MRT-Untersuchungen bedarf es deshalb entsprechend weitergebildeter und kontinuierlich fortgebildeter Fachärztinnen und Fachärzte.

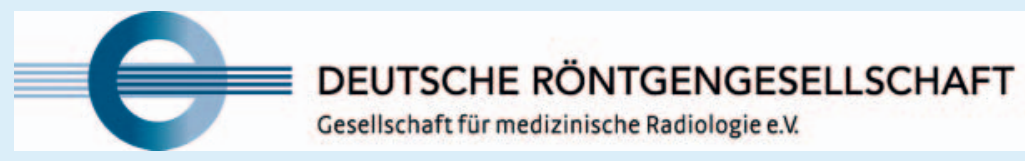

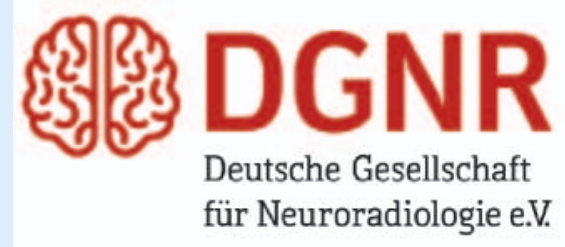

pier der Deutschen Röntgengesellschaft (DRG), der Deutschen Gesellschaft für Neuroradiologie (DGNR) und der Gesellschaft für Pädiatrische Radiologie (GPR) zu den fachlichen Anforderungen an Durchfüh-
Was dabei für die Bereiche Qualitätssicherung und Leistungserbringung unbedingt erforderlich ist, zeigt die ausführliche und wissenschaftlich fundierte Stellungnahme zur bildgebenden Methode MRT von 3 Fachgesellschaften, unter anderem der Deutschen Röntgengesellschaft: „Positionspa-

\section{GPR Gesellschaft für Pădiatrische Radiologie e.V.}

rung und Befundung von MRT-Untersuchungen außerhalb des Fachgebietes Radiologie“.

Sie möchten mehr erfahren? Das vollständige Positionspapier finden Sie unter: www.drg.de > wichtige Beiträge 